\title{
Space magnetometer based on an anisotropic magnetoresistive hybrid sensor
}

\author{
P. Brown, ${ }^{1, a)}$ B. J. Whiteside, ${ }^{1}$ T. J. Beek, ${ }^{1}$ P. Fox, ${ }^{1}$ T. S. Horbury, ${ }^{1}$ T. M. Oddy, ${ }^{1}$ M. O. Archer, ${ }^{1}$ J. P. \\ Eastwood, ${ }^{1}$ D. Sanz-Hernández, ${ }^{2}$ J. G. Sample, ${ }^{3}$ E. Cupido, ${ }^{1}$ H. O'Brien, ${ }^{1}$ and C. M. Carr ${ }^{1}$ \\ ${ }^{1)}$ Blackett Laboratory, Imperial College London, London SW7 $2 B W$, United Kingdom \\ 2) EMM-NANO Consortium, K.U. Leuven, 3000 Leuven, Belgium \\ ${ }^{3)}$ Space Sciences Laboratory, University of California, Berkeley, CA 94720
}

(Dated: 12 June 2014)

We report on the design and development of a low resource, dual sensor vector magnetometer for space science applications on very small spacecraft. It is based on a hybrid device combining an orthogonal triad of commercial anisotropic magnetoresistive (AMR) sensors with a totem pole H-Bridge drive on ceramic substrate. The drive enables AMR operation in the more sensitive flipped mode and this is achieved without the need for current spike transmission down a sensor ultra-light harness. The magnetometer has sensitivity of better than $3 \mathrm{nT}$ in a $0-10 \mathrm{~Hz}$ band and a total mass of $104 \mathrm{~g}$. Three instruments have been launched as part of the TRIO-CINEMA space weather mission and a next generation implementation will fly on a solar sail technology demonstrator due for launch in 2016.

\section{INTRODUCTION}

Magnetometers based on magnetoresistance (MR) have become increasingly popular in recent years ${ }^{1,2}$ due to the wide availability of sensor devices and low cost commercial off the shelf (COTS) chips. ${ }^{3}$ Applications such as space are increasingly utilising magnetoresistive technology, due to the very low mass and volume of the sensors (COTS MR sensors typically have a mass less than $1 \mathrm{~g})$. Typical space applications are in the areas of on-board attitude orbit control systems (AOCS) particularly on nano and picosats ${ }^{4}$. Several magnetoresistive magnetometers have been launched into space in recent years in place of more sensitive but higher resource fluxgates. ${ }^{5}$

The space applications requiring the highest performance magnetometers are scientific measurements for plasma and planetary missions where sub-nT accuracy is often required, ${ }^{6}$ in comparison to AOCS magnetometers, where $0.1 \mu \mathrm{T}$ uncertainty is typically sufficient. ${ }^{7}$ The key performance requirements for DC space science magnetometers are high sensitivity, low noise, excellent linearity over a wide dynamic range (e.g. $100 \mathrm{pT}$ to up to $50,000 \mathrm{nT}$ ) and extremely small temperature drift of the sensor offset and gain. ${ }^{8,9}$ These properties need to be achieved while minimising the instrument resources (mass, power, volume) and the increasingly easy availability (and low cost) of MR sensors means that efforts to develop higher sensitivity MR magnetometers capable of detecting low amplitude science signals and competing with heritage fluxgates designs on vehicles such as planetary landers and nanosats has become increasingly popular. ${ }^{10,11}$

Typically older technology Anisotropic Magnetoresistive (AMR) permalloy sensors have been the preferred

\footnotetext{
a) patrick.brown@imperial.ac.uk
}

sensing option for dc MR magnetometers. This is for a number of reasons: (i) they possess the highest detectivity at low frequencies $(0-10 \mathrm{~Hz})$ in comparison with other MR devices such as Giant or Tunneling magnetoresistance; ${ }^{12}$ (ii) they produce a bipolar output permitting measurement of positive and negative fields with a single device; ${ }^{13}$ (iii) as the sensor is anisotropic it is possible to modulate the direction of the easy axis and this provides a mechanism to track and compensate for the sensor bridge offset and maintain the sensor in its lowest noise operating state even in the presence of a large background ambient field. ${ }^{14}$ The modulation (commonly referred to as flipping) is achieved by driving bipolar current signals into on-chip coils that are aligned with the easy axis direction of the sensing element and integrated into the manufactured AMR package. ${ }^{15}$

In order to measure low-amplitude science signals aboard spacecraft (i.e. $<100 \mathrm{nT}$ ) it is necessary to accommodate the magnetometer sensor on a deployable boom. This positions the sensor away from spacecraft magnetic fields produced by ferromagnetic material and current loops. To achieve the highest performance an AMR magnetometer will be configured as a null detector in a control loop featuring a constant flip drive at frequencies of a few hundred Hz. A vector magnetometer instrument is thus composed of a sensing triad of AMR sensors connected by harness to a spacecraft mounted electronics unit which houses the magnetometer drive, sense and feedback functions.

Depending on the specific AMR sensor, flipping current amplitudes of up to $3 \mathrm{~A}$ for several microseconds can be required in order to achieve a full $180^{\circ}$ rotation of the easy axis. ${ }^{15}$ In the case of picosats and nanosats this presents a problem as the sensor boom harness must be made using high gauge wire in order to keep the mass as low as possible. Therefore, line losses in the harness will attenuate the flipping current and in the case of a $1 \mathrm{~m}$ harness the attenuation is such that it effectively eliminates all the benefits (such as low noise and offset) of operat- 
ing the sensor in a driven closed loop. A novel method of dealing with this problem is to migrate the flip current generation off the platform electronics and co-locate it with the AMR sensors. The current pulse is generated at the sensor, eliminating the need to overdrive a higher current pulse trains from the platform down lossy cables. This technique saves power and permits the use of an arbitrarily long boom as it decouples the efficiency of the flipping drive from the harness length.

In this article we present a compact dual-sensor vector magnetometer featuring an orthogonal AMR sensor triad combined with a hybrid flipping driver circuit. The magnetometer has been developed for a three CubeSat constellation mission called TRIO-CINEMA (CubeSat for Ions Electrons and Neutrals) ${ }^{16,17}$ where there was a specific requirement to accommodate a magnetometer sensor on an ultra-light $1 \mathrm{~m}$ stacer boom with a maximum harness mass of only $10 \mathrm{~g}$. The combined sensor and driver is implemented on a ceramic tile and the only flipping related signals transmitted are a voltage bias and drive clock. The sensor operates in the optimum low noise state for connecting harness of any length. The magnetometer resolution is better than $3 \mathrm{nT}$ in a $0-10 \mathrm{~Hz}$ band for a measurement range $\pm 57,500 \mathrm{nT}$ and a total instrument mass of only $104 \mathrm{~g}$.

\section{MAGNETOMETER DESIGN}

The magnetometer, called MAGIC (MAGnetometer from Imperial College), is composed of two functional blocks: the sensor head comprising the hybrid magnetic sensing element, implemented using three single axis Honeywell HMC1001 ${ }^{15}$ AMR sensors, and an electronics card housing the magnetometer control electronics. Each single axis sensor is operated in a driven closed loop with first harmonic detection of the DC magnetic field $^{18}$ and the feedback voltage sampled by an 8 channel 24 bit delta-sigma Analogue to Digital Converter (ADC). The hybrid sensor connects to the control electronics via a $1 \mathrm{~m}$ ultra-low mass harness (10 g without connector) comprised of 18 lines.

\section{A. Hybrid AMR Sensor}

A schematic of the hybrid design is shown in Fig. 1. It is constructed on a single thick film low temperature cofired ceramic (LTCC) tile and consists of three discrete single axis AMR sensors arranged in an orthogonal triad, $\mathrm{N}$ and $\mathrm{P}$ channel power metal oxide semiconductor field effect transistors (MOSFET) (FDN357N and FDT458P), inverting and non-inverting MOSFET drivers (TC4429 and MIC4420) and some discrete passives (thermistor and capacitors). The MOSFETs and the drivers are implemented as wire bonded die and covered in a glob top.

The HMC1001 was selected due to its superior noise floor at low frequency. ${ }^{12}$ It is comprised of a four element
Wheatstone bridge with each half bridge Permalloy element having opposite magnetizations which doubles the sensor output. Each element is patterned with Barber pole shunts to shift the operating point to the most linear region of the AMR transfer function. ${ }^{19}$ The bridge bias, return and the differential bridge output constitute four of the 8 pins on the device. The other four pins connect to a set-reset strap (parallel to the sensor easy axis) and offset strap (parallel to the sensor sensing (hard) axis), these are connected to the co-located drive pulse and remotely located feedback current generator respectively.

The drive circuit is implemented on the hybrid in a totem pole $\mathrm{H}$-Bridge configuration, formed from the dual P-N MOSFET and driver die. Bi-polar current pulses of $4 \mathrm{~A}$ are generated at $512 \mathrm{~Hz}$ and these are coupled into the $1.5 \Omega$ set-reset straps (flip coils) of the three AMR sensors which are connected in series. The pulse train switches the easy axis orientation of the AMR elements in the bridge through $180^{\circ}$ which causes the sensor output to change polarity resulting in a square wave output at the drive frequency. The capacitors $(15 \mu \mathrm{F}$ tantalum and two $220 \mathrm{nF}$ ceramics) were screened for magnetic signature (palladium-silver barrier were chosen in preference to nickel) and low electrical series resistance (ESR). The thermistor (Heraeus C220 Pt 100) was also verified nonmagnetic by screening.

The generation of the flipping current pulses locally on the hybrid means the only signals relating to the sensor drive function transmitted down the harness are a $13 \mathrm{~V}$ bias for the H-Bridge and the $512 \mathrm{~Hz}$ drive clock, which is generated on the main electronics card. This permits the use of extremely high gauge wire (36 AWG magnet wire was mandated for TRIO-CINEMA) while maintaining the ability to deliver short high current drive spikes into the set-reset straps.

The harness is made up of 18 enamel insulated wires wrapped in an outer jacket made from Arracon braid which although very slightly magnetic is highly flexible and allows for a very small bending radius (below $1 \mathrm{~cm}$ ). In the region close to the sensor it was replaced with a slightly heavier non-magnetic silver over copper braid. As well as the bias and clock for the drive the 18 constituent wires in the harness are used for the $5 \mathrm{~V}$ bias for the AMR bridges, the differential sense and feedback lines for the $\mathrm{Bx}, \mathrm{By}, \mathrm{Bz}$ components and the thermistor line.

The hybrid has dimensions of $2 \mathrm{~cm} \times 2 \mathrm{~cm} \times 0.5 \mathrm{~cm}$ and a mass of $<1 \mathrm{~g}$. All component parts used in the hybrid are commercial grade, however both the semiconductor die parts and capacitors are available as radiation hardened space qualified versions. A photo displaying the two sides of the hybrid showing the orthogonal arrangement of the AMR chips is shown in Fig. 2. In the flight configuration the hybrid is housed inside a $2 \mathrm{~cm} \times$ $2 \mathrm{~cm} \times 2 \mathrm{~cm}$ aluminium casing and held in a fixed position through the use of resin spacers. The whole head is potted in epoxy to provide both mechanical stability and to increase the thermal inertia to limit rapid temperature 


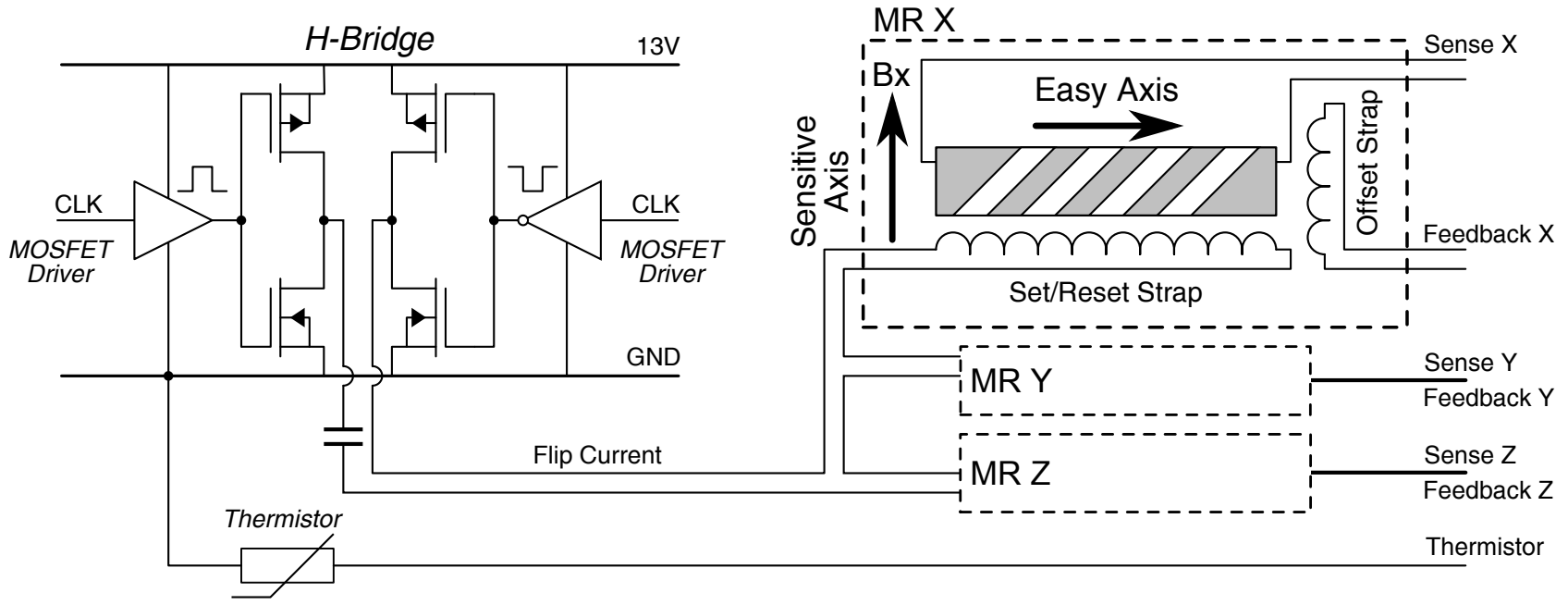

FIG. 1. A schematic of the electronics contained within the hybrid sensor. The sense, feedback, power and CLK signals originate from the electronics card. The H-Bridge circuit is implemented as wire bonded die and the MRs are three discrete packages that form a vector triad.

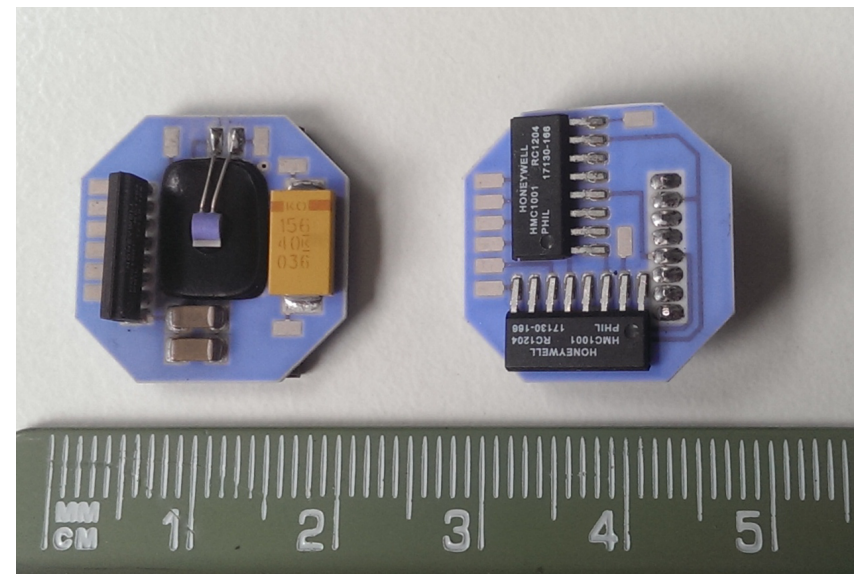

FIG. 2. Image of the hybrid chip from above and below. On the left hand side is the out of plane AMR sensor $(\mathrm{Bz})$, the passives and the black glob-top that covers the MOSFET die. The right hand side shows the in-plane AMR sensors (Bx,By)

changes inside the MR sensors (e.g. as the sensor goes in and out of eclipse).

\section{B. Control Electronics}

The electronics card houses the drive and sense analogue electronics and a 24 bit ADC (TI ADS1217). A schematic of the control electronics is shown in Fig. 3. Both analogue and digital electronics operate off a single ended $5 \mathrm{~V}$ supply and a $17 \mathrm{~V}$ line (which is dropped to $13 \mathrm{~V}$ ) provides the bias for the H-Bridge flipping. There are six individual control loops in total, three for the three axis hybrid and three for a second orthogonal sensor triad of HMC1001s that are housed on the electronics card itself (and known as the inboard sensor). This inboard sensor was included for the purpose of redundancy in case of failure of the outboard hybrid sensor or harness connection. Each individual loop for the outboard sensor axes is composed of a differential low-noise pre-amp which amplifies the low amplitude flipped signal in parallel with an analogue offset compensator to remove the bridge offset as described previously. ${ }^{18}$ The DC centred signal is then demodulated and fed to an integrator which is used to control a feedback current via an op-amp, the output current of the op-amp flows down the harness to the offset strap which thereby closes the loop. The output of the integrator is directly proportional to the field component along the sensitive axis of the HMC1001 and this is fed into a channel of the ADC via a simple antialiasing filter $(-3 \mathrm{~dB} 16 \mathrm{~Hz})$. All op-amps are OP-484s from Analog Devices. The ADC includes a built-in 8 channel multiplexer and this is used to switch the input between the output filter of the 6 magnetometer channels (outboard and inboard). The other two channels are used to monitor the $5 \mathrm{~V}$ bus voltage (as it moves so does the $2.5 \mathrm{~V} \mathrm{ADC}$ reference) and the hybrid sensor thermistor reading, (which is also conditioned using an OP-484).

The ADS1217 is a second order delta-sigma modulator. The modulator sample rate is set by the Master Clock divided by 128 . Off board communications are performed through the ADC using a Serial Peripheral Interface (SPI). Code was developed on a Teensy microcontroller (based on an Atmel ATmega32U4 microprocessor) and used to switch the ADC channel, sample the input and apply an in-built digital filter which is configured over the SPI link. Several filters are available and a "sinc" 3 " filter was chosen in a trade-off between settling time and bandwidth as it offers the lowest noise and highest effective number of bits (ENOB) at the expense of settling time, which in turn limits the vector sample 


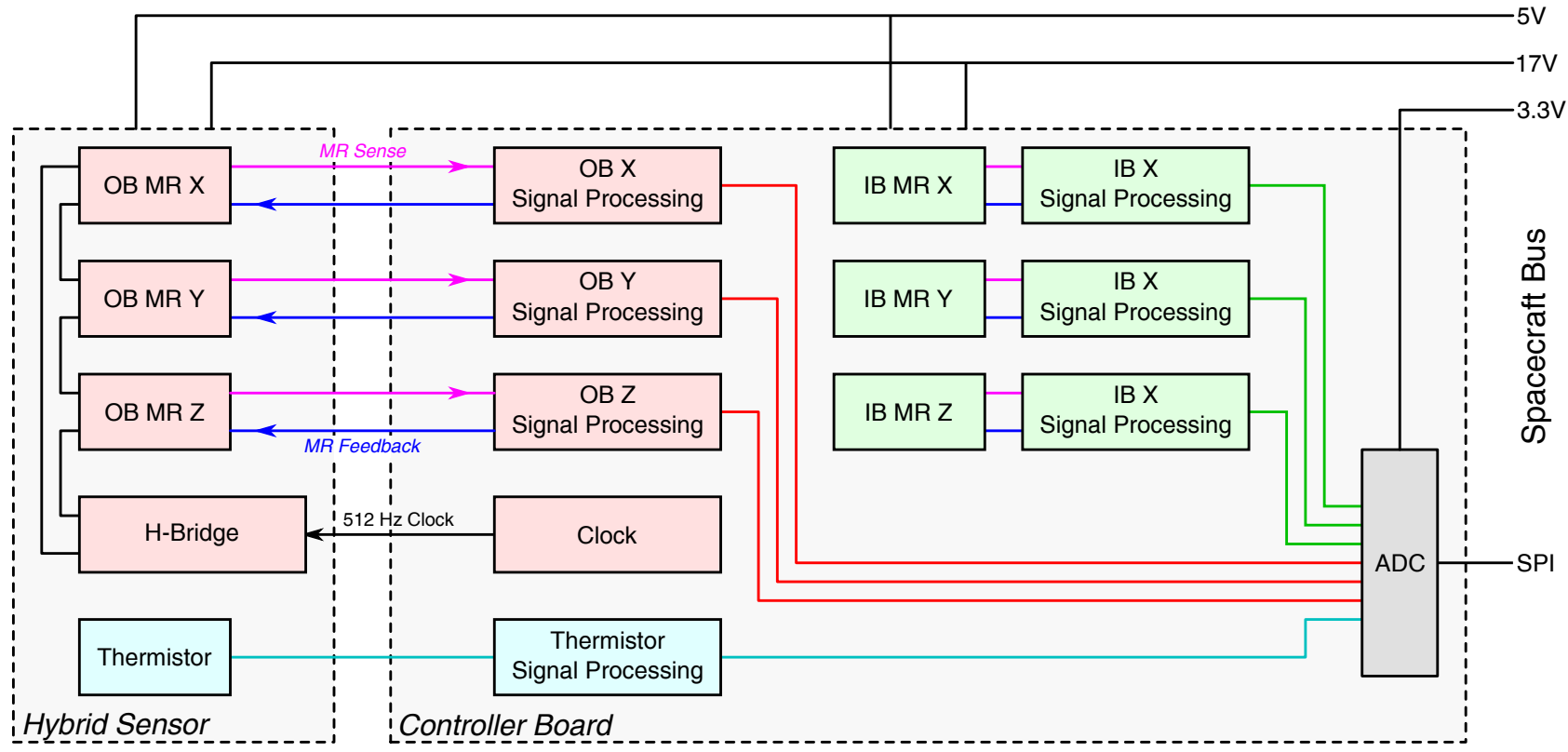

FIG. 3. Magnetometer block diagram. The hybrid sensor is connected to the controller board via a $1 \mathrm{~m}$ harness. The controller board contains the signal processing control for the hybrid, the complete inboard vector magnetometer and ADC.

rate. For the TRIO-CINEMA implementation multiple vector cadences were required:

- a low sensitivity, low power Attitude Mode $(4 \mathrm{~Hz})$ sampling the inboard sensor

- a high sensitivity, high power, Science Mode $(8 \mathrm{~Hz})$ sampling the outboard hybrid sensor

- a diagnostic, high power 'Gradiometer Mode' (also $8 \mathrm{~Hz}$ ) sampling the outboard hybrid sensor and inboard sensor at the same rate.

This use of different cadences meant the on-board digital filter has to be configured differently for each instrument mode.

The filter is configured through a programmable onchip register storing the decimation setting, calculated according to

$$
F_{D e c}=0.95\left(\frac{f_{\text {osc }} \times \Delta t}{128}\right)
$$

where $F_{D e c}$ corresponds to the filter decimation, $f_{\text {osc }}$ is the ADC master clock frequency $\left(2^{22} \mathrm{~Hz}\right)$ and $\Delta t$ is the time between component samples; the 128 is the deltasigma ADC modulation factor and the 0.95 allows for $5 \%$ tolerance on the sampling to ensure the ADC settles before acquiring the next sample. The $\Delta t$ corresponds to one quarter of the transmitted $8 \mathrm{~Hz}$ vector sample period in Science Mode (one quarter because each vector packet corresponds to the individual $\mathrm{Bx}, \mathrm{By}, \mathrm{Bz}$, components sampled consecutively followed by a temperature reading with a $31.25 \mathrm{~ms}$ sample interval). Attitude mode sampling is configured differently; as it has a smaller $4 \mathrm{~Hz}$ cadence it was feared that for the crucial period where the CubeSat would use the Attitude Mode to autonomously de-tumble and spin up after release from the carrier spacecraft, the component samples of each attitude vector should have the minimal phase delay possible. Consequently the phase delay between component samples is actually much smaller than a nominal one quarter of the $4 \mathrm{~Hz}$ vector rate $(7.825 \mathrm{~ms}$ as opposed to $62.5 \mathrm{~ms}$ is used) with a much longer lag between successive vectors than in Science Mode where the overall transmitted vector rate is higher but the phase lag between each individual components of each vector is larger and all samples are equally spaced in time.

In the case of Science Mode the decimation is therefore 324 (for a $\Delta t$ of $31.25 \mathrm{~ms}$ ) and in Attitude Mode it is 80 (for a $\Delta t$ of $7.8125 \mathrm{~ms}$ ). The as-used value of $F_{D e c}$ is taken as a multiple of 4 nearest to the calculated theoretical value as this optimises the data-ready time period within the ADC. ${ }^{20}$

The ADC also includes a number of general purpose IO lines, four of which are used, and these can be set via SPI command. These bits were used to configure hardware elements of the electronics card (such as switch on-off of the flipping drive etc) in accordance with a set of defined tele-commands.

While the magnetometer was initially developed for low field applications such as planetary and lunar landers (target ambient field $<5000 \mathrm{nT}$ ) as the first flight opportunity was to be the TRIO-CINEMA CubeSat launching to Low Earth Orbit (LEO), a large dynamic range was required. A range of $\pm 57,500 \mathrm{nT}$ was implemented with $0 \mathrm{nT}$ corresponding to $2.5 \mathrm{~V}$ input to the ADC channel, which corresponds to a nominal scale factor of $23,000 \mathrm{nT} / \mathrm{V}$. This caused some problems for the feed- 


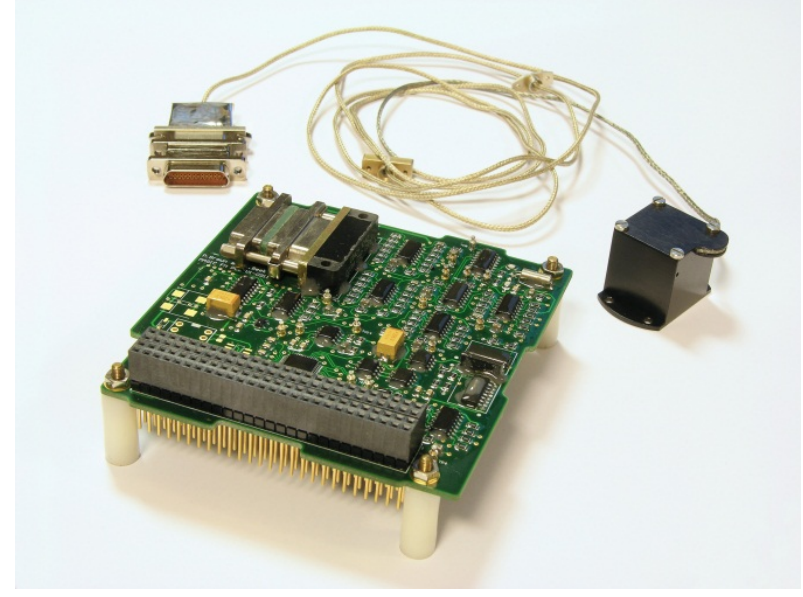

FIG. 4. Photo of one of the Trio-CINEMA flight instruments.

back and overall loop stability which is discussed further in Section IV.

Table I details the basic instrument properties and parameters including the hardware configuration and decimation for the different instrument modes The magnetometer is very low mass (total mass of the entire instrument is $104 \mathrm{~g}$ including $1 \mathrm{~m}$ harness) and power consumption varies within the range $(0.14 \mathrm{~W}$ to $0.5 \mathrm{~W})$ according to the selected mode. Fig. 4 shows a photo of the entire instrument.

TABLE I. Instrument Properties

(a) Basic Instrument Properties

\begin{tabular}{ll}
\hline \hline Mass & $33 \mathrm{~g}$ (potted sensor and $1 \mathrm{~m}$ harness) \\
& $71 \mathrm{~g}$ (electronics) \\
& $104 \mathrm{~g}$ (total) \\
& Sensor head $10 \mathrm{~cm}^{3}$ \\
Volume & Electronics $173 \mathrm{~cm}^{3}(9 \times 9.6 \times 2 \mathrm{~cm})$ \\
& $\pm 57,500 \mathrm{nT}$ \\
Range & $0.2 \mathrm{nT}(19 \mathrm{bit}$ transmission) \\
Digital Resolution & $16 \mathrm{~Hz}$ \\
-3dB point & $150 \mathrm{pT} \mathrm{Hz}-1 / 2$ (above $1 \mathrm{~Hz})$ \\
Noise Density & $-50{ }^{\circ} \mathrm{C}$ to $+60^{\circ} \mathrm{C}$ (electronics) \\
Operational Temperature & $-120^{\circ} \mathrm{C}$ to $+80^{\circ} \mathrm{C}$ (sensor) \\
&
\end{tabular}

(b) Mode specific properties. IB $=$ Inboard, OB = Outboard

\begin{tabular}{llll}
\hline \hline Instrument Mode & Attitude & Science & Gradiometer \\
Operational Sensor & IB Only & OB Only & IB and OB \\
Flip Frequency $(\mathrm{Hz})$ & None & 512 & $512(\mathrm{OB})$ \\
Sensitivity $(\mathrm{nT})$ & 10 & 2 & $\mathrm{IB}=10, \mathrm{OB}=2$ \\
Cadence $\left(\right.$ vectors $\left.\mathrm{S}^{-1}\right)$ & 4 & 8 & 8 from both \\
ADC Sample Rate $(\mathrm{Hz})$ & 128 & 32 & 64 \\
Decimation $\left(F_{\text {Dec }}\right)$ & 80 & 324 & 160 \\
Bus Rails $(\mathrm{V})$ & $3.3,5$ & $17,5,3.3$ & $17,5,3.3$ \\
Power $(\mathrm{mW})$ & 140 & 425 & 560 \\
\hline \hline
\end{tabular}

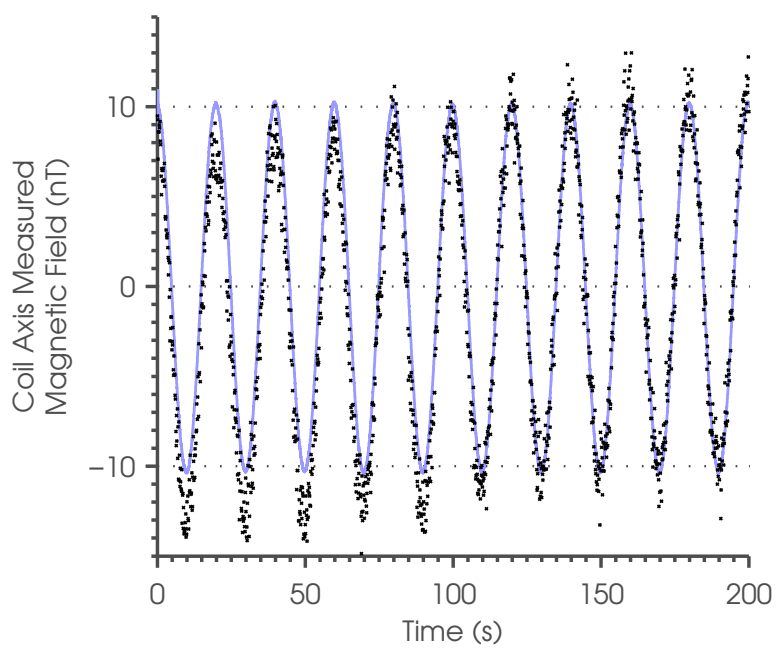

FIG. 5. A $0.05 \mathrm{~Hz}$ sinusoidal magnetic field applied along one axis of the hybrid with an amplitude of $10 \mathrm{nT}$. The hybrid output (dots) is compared to a reference fluxgate measurement (line).

\section{MAGNETOMETER CALIBRATION AND PERFORMANCE}

Test and calibration of the magnetometer has been performed for the three TRIO-CINEMA flight units, concentrating on characterization of the outboard hybrid sensor operation in Science Mode. Noise, offset and offset temperature drift have been measured in the laboratory using a three layer mu-metal shield tank with an integrated single axis solenoid stimulus coil and a highly accurate three axis reference fluxgate magnetometer. ${ }^{21}$

The sensitivity of the magnetometer is shown in Fig. 5 for one axis under the application of a $0.05 \mathrm{~Hz}$ sine wave. We can see that the $10 \mathrm{nT}$ sine wave is clearly resolved. A power spectral density of the magnetometer digital output in Science Mode for all three axes is plotted in Fig. 6. The detectivity of the three axes averaged around $1 \mathrm{~Hz}$ are as follows: $\mathrm{Bx}\left(324 \mathrm{pT} \mathrm{Hz}^{-1 / 2}\right)$, By $\left(339 \mathrm{pT} \mathrm{Hz}^{-1 / 2}\right)$ and $\mathrm{Bz}\left(312 \mathrm{pT} \mathrm{Hz}^{-1 / 2}\right)$. The magnetometer noise density at $1 \mathrm{~Hz}$ is greater than that previously reported for our single axis magnetometer design. ${ }^{18} \mathrm{~A}$ noise test was subsequently performed on one axis of the hybrid output at the input to the ADC and this is plotted in the inset of Fig. 6 together with that of the reference fluxgate. The noise density of the $\mathrm{MR}$ output at $1 \mathrm{~Hz}$ is $38 \mathrm{pT} \mathrm{Hz}^{-1 / 2}$ compared to $8 \mathrm{pT} \mathrm{Hz}^{-1 / 2}$ for the fluxgate. This suggests that the digitization of the analogue output is contributing significantly to the overall magnetometer noise level. This is an area to be targeted for improvement in future designs.

Longer term drift of the hybrid sensor is shown in Fig. 7 where the hybrid MR sensor is fitted within the mumetal shield and operated in the laboratory over several days. We can see a slow periodic variation in the time series of all three axes, the drift is assumed to be offset 


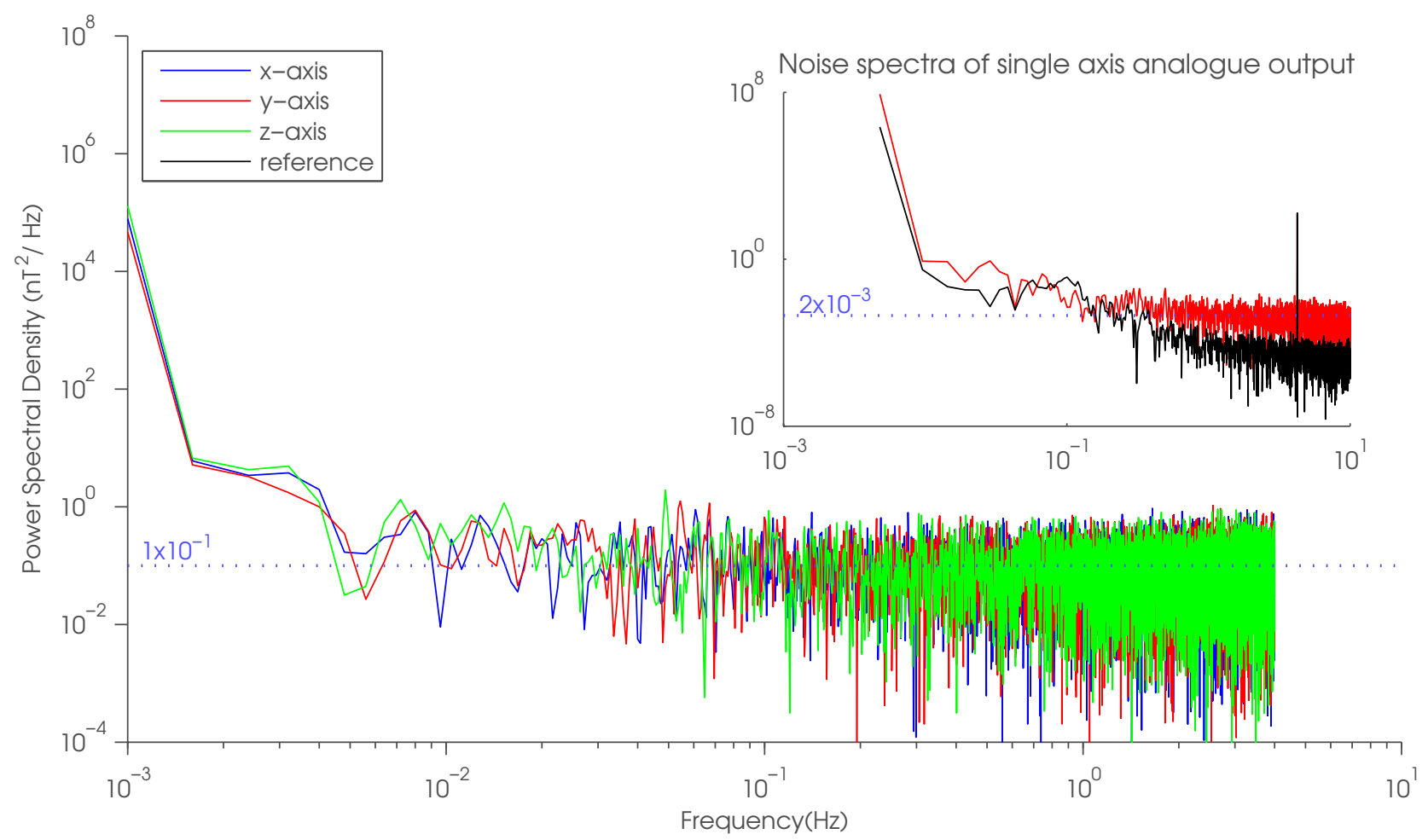

FIG. 6. Power spectral density of the three axes from the outboard sensor in Science Mode. The inlay is a power spectral density of the y-axis with the same setup but before digitisation together with a collocated with a reference fluxgate sensor, the noise of the y-axis is significantly reduced.

drift with temperature.

Inter axis misalignments and gains have been determined using a rotation table at Imperial's non-magnetic calibration facility located in Staffordshire, UK. The sensor calibration matrix transforms the measured magnetic field vector from the non-orthogonal sensor frame into an orthogonalised reference frame in accordance with the following equation

$$
\mathbf{B}_{c}=C_{m}\left(\mathbf{B}_{m}-\mathbf{O}\right)
$$

where $\mathbf{B}_{m}$ is the measured vector, $\mathbf{O}$ is measured offset vector, $C_{m}$ is a $3 \times 3$ calibration matrix and $\mathbf{B}_{c}$ is the calibrated vector. The off diagonal elements of $C_{m}$ corresponding to the 6 inter-axis misalignment and diagonal elements correspond to the gain values of the individual sensor $\mathrm{x}, \mathrm{y}, \mathrm{z}$ axes. The calibration matrix has been determined as follows: the hybrid sensor and reference fluxgate are mounted onto Tufnel jigs that have a high mechanical tolerance. The sensor offset vector, $\mathbf{O}$, is measured using a shielding can fitted with a mounting track for the two jigs. After this the reference fluxgate is fixed to the centre of the rotation table and the average of Earths magnetic field is measured through a $180^{\circ}$ rotation. The reference is then replaced with the hybrid sensor and rotated through 6 different $90^{\circ}$ rotations. The jig ensures an accurate $90^{\circ}$ rotation when rotating the axis that is not in the plane of the rotation table. The hybrid data is then fitted by an iterative least squares algorithm to

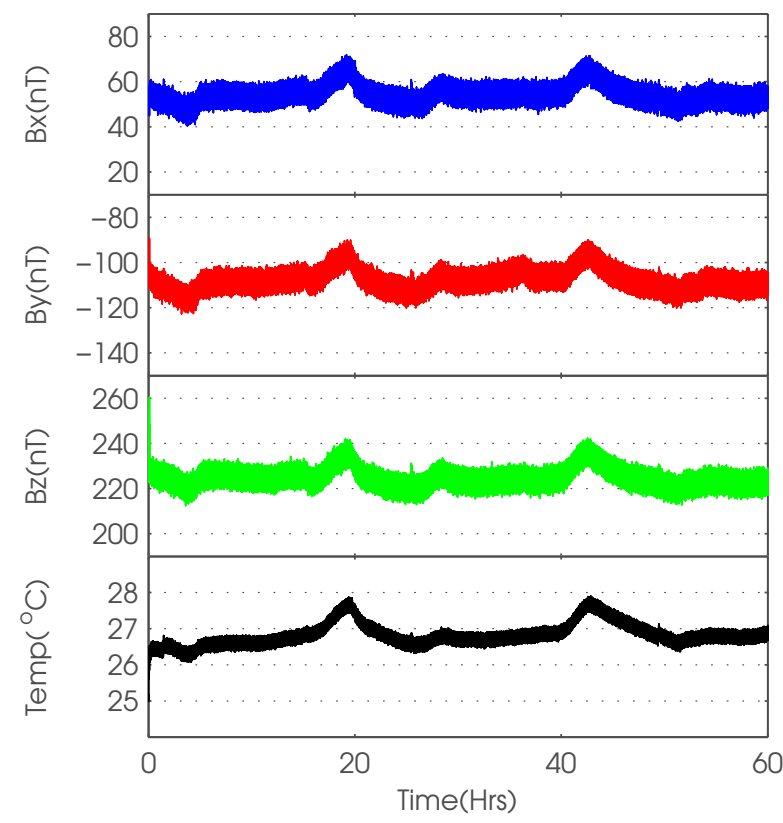

FIG. 7. Sensor output drift as a function of time with the temperature variation measured via the hybrid thermistor. Peak-to-peak noise is higher due to large EMC disturbance from thermal chamber. The overall trend however is still clearly visible. 
the fluxgate data under the assumption that the ambient field has been constant for the duration of the measurement of the two sensors. Once the matrix $C_{m}$ has been calculated a second set of 6 rotation measurements are taken with the hybrid and the calculated matrix is applied to the data to ensure that the calibrated field is within an acceptable error to that of the fluxgate. The calibration procedure is repeated if the residual error is greater than $1 \%$.

Six magnetometers have been calibrated to date and the calibration results are shown in Table II. In each case the sensor has been potted inside its aluminium housing so the mechanical orientations of the three individual HMC1001 sensors are rigidly fixed. As they are only visually aligned they do not form a perfect orthogonal triad and deviations of up to a few degrees can occur, however is not a problem as long as the misalignments are known from the ground calibration. Existing methods of in-flight determination of calibration parameters use, for example, Fourier based techniques applied to spin stabilised spacecraft ${ }^{22}$ and the International Geomagnetic Reference Field (IGRF) for LEO. ${ }^{23}$ Such techniques may be modified for application to TRIO-CINEMA in principle.

It can be seen from Table II that the offsets are in the range of a few hundred nT up to $2000 \mathrm{nT}$. This offset is comprised of electrical and sense effects. There are several different sources of electrical errors that contribute to the offset: these are caused by non-ideal op-amp behavior, small resistor mismatch and input bias currents. The Wheatstone bridge offset and demodulation offset are almost negligible due to the active compensation provided by a feedback integrator at the preamplifier stage. The sense effects may be due to stray magnetic fields within the hybrid as a result of the flipping current. The sense offsets can be nulled using additional current injection into the offset strap but these are still susceptible to temperature effects. Options to improve the overall offset performance and stability are discussed is section IV.

Fig. 8 plots the magnetometer output and temperature of the sensor in a low field shielding tank that has been installed within a thermal chamber. The temperature was varied between $+54{ }^{\circ} \mathrm{C}$ and $+24^{\circ} \mathrm{C}$. We can see that the $\mathrm{Z}$ axis has the worst drift of $16.6 \mathrm{nT}^{\circ} \mathrm{C}^{-1}$ and the $\mathrm{X}$ axis has the smallest drift of $1.86 \mathrm{nT}^{\circ} \mathrm{C}^{-1}$. As the sensors are inside a shielding can we associate the observed drift with a changing sensor offset, however there will be a contribution from the change in the ambient field inside the can due to the temperature change in the surrounding mu-metal in addition to the potential effect of the magnetically noisy thermal chamber. The observed drift is not as good as that previously reported for the case of using standalone HMC1001 sensors driven from an electronics card $^{18}$ and further investigation is required to determine if there is an increased thermal susceptibility due to use of the hybrid sensor design.

One model of the magnetometer has also undergone

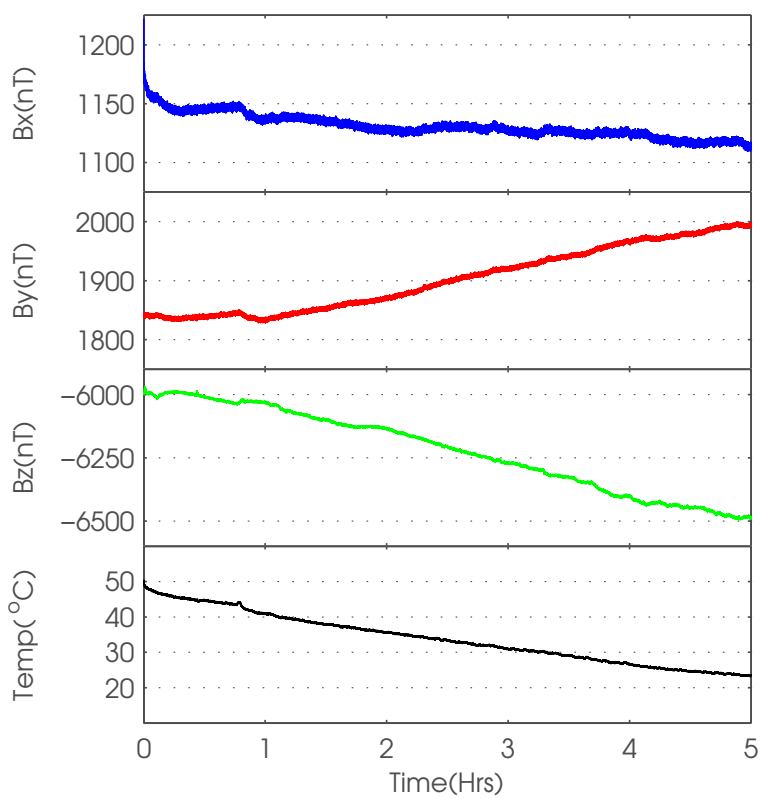

FIG. 8. Variation of sensor offset with temperature.

formal qualification testing: mechanical vibration testing at $23.15 g_{r m s}$ between 20 and $2000 \mathrm{~Hz}$; and thermal vacuum (as part of one of the CINEMA CubeSats) in the range -20 to $+35^{\circ} \mathrm{C}$, with a 2 hour soak time. The instrument remained fully functional after both tests. In addition the hybrid sensor has undergone total ionising dose (TID) radiation testing up to $105 \mathrm{kRad}$ and maintained nominal functionality. ${ }^{24}$

\section{DISCUSSION}

While the magnetometer has very good sensitivity in the range $0-20 \mathrm{~Hz}$ the major performance limitation of the design at present is the offset and particularly the offset drift. The issue is partially mitigated if the ambient field of the target flight environment is substantially less than that in LEO and this will be the case for next flight implementation of the hybrid magnetometer, (the Sunjammer mission to Lagrange point 1 in the solar wind) where a maximum range of only $\pm 500 \mathrm{nT}$ is required in the science phase. ${ }^{25}$ In fact, the original pre-hybrid magnetometer design was targeted at much lower field ranges than Earths field and this caused a problem when the operating range was scaled up to $\pm 57,500 \mathrm{nT}$ for the TRIO-CINEMA hybrid design. It was found necessary to reduce the integrating feedback capacitance and add further gain at the integrator stage of each component channel in order to limit the clipping and non-linearity while maintaining the needed bandwidth. This was a consequence of the relative large current $(29 \mathrm{~mA})$ required by the HMC1001 offset strap to null a 57,500 nT field and the inability of the op-amps used in the voltage to current converter to drive more than a $5 \mathrm{~mA}$ current. Therefore 
TABLE II. Hybrid Sensor Calibration Results for several of the MAGIC instruments. Models FM2 and FM3 are currently in-flight, Models FM4 is a flight ready spare, EM is an engineering models. ND is Not Determined.

\begin{tabular}{|c|c|c|c|c|c|c|c|c|c|c|c|c|c|c|c|}
\hline \multirow{2}{*}{$\begin{array}{l}\text { Cal. } \\
\text { Parameter } \\
\text { Magic } \\
\text { Model }\end{array}$} & \multicolumn{3}{|c|}{ Gains (nT V $\left.\mathbf{V}^{-1}\right)$} & \multicolumn{3}{|c|}{ Offsets (nT) } & \multicolumn{2}{|c|}{ Calibration } & \multicolumn{4}{|c|}{ Matrix Elements $\left(C_{i j}\right)$} & \multirow[b]{2}{*}{$C_{31}$} & \multirow[b]{2}{*}{$C_{32}$} & \multirow[b]{2}{*}{$C_{33}$} \\
\hline & $\mathrm{G}_{x}$ & $\mathrm{G}_{y}$ & $\mathrm{G}_{z}$ & $O_{x}$ & $O_{y}$ & $O_{z}$ & $C_{11}$ & $C_{12}$ & $C_{13}$ & $C_{21}$ & $C_{22}$ & $C_{23}$ & & & \\
\hline FM2 & 23205.84 & 24782.01 & 24151.03 & 239 & -705 & -426 & ND & ND & ND & ND & ND & ND & ND & ND & ND \\
\hline FM3 & 22634.78 & 22908.70 & 23179.38 & -497 & -102 & -230 & ND & ND & ND & ND & ND & ND & ND & ND & ND \\
\hline$F M_{4}$ & 20757.11 & 20256.54 & 20300.88 & 912 & 255 & 1593 & 0.902 & 0.055 & 0.027 & -0.009 & 0.881 & 0.008 & 0.027 & 0.015 & 0.883 \\
\hline FM5 & 22155.90 & 21781.69 & 21310.42 & 130 & 584 & -1514 & 0.963 & 0.013 & -0.018 & -0.011 & 0.947 & -0.014 & 0.001 & -0.013 & 0.927 \\
\hline ЕМЗ & 21089.62 & 22032.57 & 24242.12 & 232 & 70 & 811 & 0.962 & -0.012 & -0.024 & 0.053 & 0.958 & 0.011 & 0.011 & -0.003 & 1.054 \\
\hline EM3-C & 22076.09 & 21919.00 & 20802.81 & -239 & 527 & -839 & 0.960 & -0.027 & 0.057 & 0.008 & 0.953 & -0.017 & 0.042 & -0.019 & 0.904 \\
\hline
\end{tabular}

the TRIO-CINEMA implementation effectively operates in a partial open loop configuration which will exhibit worse offset drift than a fully closed loop implementation.

Key areas of future work are to implement full current feedback drive in the Earth field range modes and improve the offset drift temperature. Improving the offset behaviour will focus on more careful matching of the overall loop gain against the bandwidth and more complete elimination of the bridge offset in the offset compensator though use of an instrumentation amplifier or individual tuning of the compensator integrators. A further area to be studied is the effect of potential stray fields in the hybrid itself caused by circulating currents in the very high gauge induction wire. This is believed to be a non-negligible effect and may require a re-routing of the ceramic tile or change in wiring configuration.

Eventually it is planned to migrate the analogue control loop into the digital domain where the demodulation is performed inside a Field Programmable Gate Array (FPGA) or Application Specific Integrated Circuit (ASIC). This may offer the best option for real time tracking and compensation of the offset drift of the flipped sensor.

Three identical hybrid magnetometers have been launched on the three TRIO-CINEMA CubeSats into LEO to date, the first launched occurred on 13th September 2012 and the second two CubeSats were launched on 3rd November 2013. Unfortunately in all three cases there was a problem with the spacecraft on-board antenna and so far there has been very limited data downloaded from the magnetometers. However it has been established that at least one of the hybrid magnetometers (from the first launch) is fully functional one year on from launch and one of the second set is confirmed operational. Fig. 9 is a plot of the three field components from the hybrid sensor lasting approximately half an orbit (46 min). The instrument was in Science Mode so the data rate is $8 \mathrm{~Hz}$, however due to spacecraft clock uncertainties we only plot the first data-point from each packet resulting in a $5 \mathrm{~s}$ cadence. Though the spacecraft was tumbling, we have been able to determine the attitude of the sen-

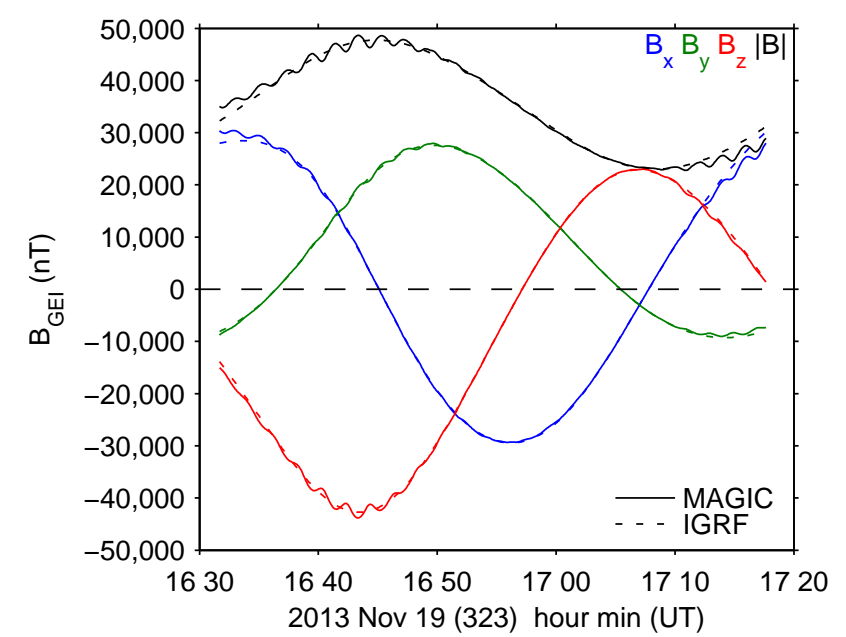

FIG. 9. Comparison of despun flight data from CINEMA 1 to IGRF. Oscillations due to residual uncertainties in despinning.

sor and calibrate the data by using the IGRF model. ${ }^{26}$ It can be seen that the magnetometer tracks the model well, with a root mean squared error of $3 \%$. The difference between the measured field and IGRF oscillate at periods of 4-10 minutes corresponding to the spin frequencies (rotation, nutation and precession) of the spacecraft, due to drifting calibration parameters over this interval. The reason for the drift is likely due to the large and varying temperatures $\left(\sim 60-80^{\circ} \mathrm{C}\right)$ experienced by CINEMA over this interval since the spacecraft had been in direct sunlight for $\sim 3$ days. Nevertheless the results show that the magnetometer design offers the prospect of ultra-low resource science $\mathbf{B}$ field measurements for future nearEarth, planetary and deep space missions.

\section{ACKNOWLEDGMENTS}

Financial support for the prototyping phase of the magnetometer has been provided by the UK Science 
Technology and Facilities Council. Development of the hybrid sensor and flight units has been funded by ESA under the General Support Technology Programme and Permanent Open Call for Technology Demonstrators. We particularly thank Dr Alain Hilgers at ESA/ESTEC for his support. We also thank the teams of undergraduate students at SSL UCB and Imperial College who worked on the design and build of the TRIO-CINEMA CubeSats or support for MAGIC test and calibration. This article is dedicated to Prof. Bob Lin, who was the inspiration and lead for the TRIO-CINEMA mission and who sadly passed away soon after the first CubeSat launch in September 2012.

${ }^{1}$ J. Lenz and S. Edelstein, "Magnetic sensors and their applications," IEEE Sensors Journal 6, 631-649 (2006).

${ }^{2}$ M. J. Caruso, "Applications of Magnetic Sensors for Low Cost Compass Systems," in IEEE Position, Location and Navigation Symposium (2000) pp. 177-184.

${ }^{3}$ A. Jander, C. Smith, and R. Schneider, "Magnetoresistive Sensors for Nondestructive Evaluation," in 10th SPIE International Symposium, Nondestructive Evaluation for Health Monitoring and Diagnostics, Conferernce 5770 (Spie, 2005) pp. 1-13.

${ }^{4}$ H. Heidt, J. Puig-Suari, A. S. Moore, S. Nakasuka, and R. J. Twiggs, "CubeSat: A new generation of picosatellite for education and industry low-cost space experimentation," in 14th Annual. AIAA/USU Conf. on Small Satellites, SSC00-V-5 (2000).

${ }^{5}$ M. Díaz-Michelena, "Small magnetic sensors for space applications." Sensors (Basel, Switzerland) 9, 2271-2288 (2009).

${ }^{6}$ L.-Y. Liu, S.-B. Jiang, T.-L. Yeh, H.-c. Yeh, J.-Y. Liu, Y.-H. Hsu, and J.-Y. Peng, "The Magneto-Resistive Magnetometer of BCU on the Tatiana-2 Satellite," Terrestrial, Atmospheric and Oceanic Sciences 23, 317-326 (2012).

${ }^{7}$ A. Balogh, C. Carr, M. H. Acuna, M. W. Dunlop, T. Beek, P. Brown, K.-H. Fornacon, E. Georgescu, K.-H. Glassmeier, T. M. Oddy, and K. Schwingenschuh, "The Cluster Magnetic Field Investigation : overview of in-flight performance and initial results," Annales Geophysicae 19, 1207-1217 (2001).

${ }^{8}$ G. M. Lerner and M. D. Shustert, "In-Flight Magnetometer Calibration and Attitude Determination for Near-Earth Spacecraft," Journal of Guidance, Control, and Dynamics 4, 518-522 (1981).

${ }^{9}$ M. H. Acuna, "Space-based magnetometers," Review of Scientific Instruments 73, 3717-3736 (2002).

${ }^{10} \mathrm{~A}$. Balogh, "Planetary Magnetic Field Measurements: Missions and Instrumentation," Space Science Reviews 152, 23-97 (2010).

${ }^{11}$ W. Magnes and M. Díaz-michelena, "Future Directions for Magnetic Sensors for Space Applications," IEEE Transactions on Magnetics 45, 4493-4498 (2009).

${ }^{12}$ N. A. Stutzke, S. E. Russek, D. P. Pappas, and M. Tondra, "Lowfrequency noise measurements on commercial magnetoresistive magnetic field sensors," Journal of Applied Physics 97, 10Q107 (2005).
${ }^{13}$ M. J. Caruso, T. Bratland, C. H. Smith, and R. Schneider, "A new perspective on magnetic field sensing," Sensors 15, 34-46 (1998).

${ }^{14}$ H. Hauser, P. Fulmek, P. Haumer, M. Vopalensky, and P. Ripka, "Flipping field and stability in anisotropic magnetoresistive sensors," Sensors and Actuators A 106, 121-125 (2003).

${ }^{15}$ Honeywell, "1- and 2-Axis Magnetic Sensors HMC1001, 1002, 1021 , 1022," Datasheet 900248 (2008).

${ }^{16}$ D.-H. Lee and R. P. Lin, "Kyung Hee University s WCU Project on Space Exploration in Lunar Orbit Research," AAPPS Bulletin 19, 18-23 (2009).

${ }^{17}$ K. Vega, D. Auslander, and D. Pankow, "Design and Modeling of an Active Attitude Control System for CubeSat Class Satellites," in AIAA Modelling and Simulation Technologies Conference, August (2009) pp. 10-13.

${ }^{18}$ P. Brown, T. Beek, C. Carr, H. O'Brien, E. Cupido, T. Oddy, and T. S. Horbury, "Magnetoresistive magnetometer for space science applications," Measurement Science and Technology 23, 059501 (2012).

${ }^{19}$ M. Endoh, N. Shimizu, H. Yoda, and N. Waoatsuki, "Highly sensitive thin film magnetoresistive sensor with good linearity," in Electronic Manufacturing Technology Symposium, 1988, Designto-Manufacturing Transfer Cycle. Fifth IEEE/CHMT International (1988) pp. 210-214.

${ }^{20} \mathrm{~J} . \mathrm{Wu}$, "Calibration Routines and Register Value Generation for the ADS1216, ADS1217 and ADS1218," Texas Instruments Application Note SBAA099, 1-10 (2003).

${ }^{21}$ C. Carr, P. Brown, T. L. Zhang, J. Gloag, T. Horbury, E. Lucek, W. Magnes, H. O'Brien, T. Oddy, U. Auster, P. Austin, O. Aydogar, A. Balogh, W. Baumjohann, T. Beek, H. Eichelberger, K.-H. Fornacon, E. Georgescu, K.-H. Glassmeier, M. Ludlam, R. Nakamura, and I. Richter, "The Double Star magnetic field investigation: instrument design, performance and highlights of the first year's observations," Annales Geophysicae 23, 2713$2732(2005)$.

${ }^{22}$ E. L. Kepko, K. K. Khurana, M. G. Kivelson, R. C. Elphic, and C. T. Russell, "Accurate Determination of Magnetic Field Gradients from Four Point Vector Measurements - Part 1," IEEE Transactions on Magnetics 32, 377-385 (1996).

${ }^{23}$ J. C. Springmann and J. W. Cutler, "Attitude-Independent Magnetometer Calibration with Time-Varying Bias," Journal of Guidance, Control, and Dynamics 35, 1080-1088 (2012).

${ }^{24}$ B. J. Whiteside, P. Brown, T. Beek, T. Oddy, T. Horbury, and C. Carr, "TID Response of a Hybrid AMR Vector Magnetometer," in To be presented at IEEE NSREC, Paris 2014 (2014).

${ }^{25}$ J. Eastwood, P. Brown, T. Oddy, B. J. Whiteside, P. Fox, N. Adeli, T. J. Beek, C. M. Carr, and N. Barnes, "Magnetic Field Measurements from a Solar Sail Platform with Space Weather Applications," in Advances in Solar Sailing (2014) pp. 185-200. ${ }^{26}$ M. O. Archer et. al., "To be submitted," (2014). 